\title{
A PROBABILISTIC APPROACH TO THE INEQUALITY ADJUSTMENT OF THE HUMAN DEVELOPMENT INDEX
}

\author{
Annibal Parracho Sant'Anna, Gilson Brito Alves Lima \\ and Luiz Octávio Gavião*
}

Received November 30, 2016 / Accepted October 23, 2017

\begin{abstract}
The Composition of Probabilistic Preferences is here applied to combine eight indicators of human development. The four components of HDI are considered together with indices of inequality in longevity, schooling, and income, and the UNDP Gender Development Index. To compose the probabilities of preference by the different criteria, a pessimistic and conservative point of view is taken. This point of view, while generating the global score, results in employing for each option the probability of not presenting the lowest evaluation according to any criterion, increasing the importance assigned in the computations to the proximity to the frontier of worst performances. Alternative rankings of the 188 countries ranked in the 2015 and the 2016 Human Development Report are obtained. Capacities allowing for interactions between up to eight components are employed. Representative profiles of ordered classes are derived from the rankings. Classifications of the countries into previously determined classes are also performed.
\end{abstract}

Keywords: Human Development Index, Composition of Probabilistic Preferences, Multiple criteria Decision analysis, Uncertainty Modelling.

\section{INTRODUCTION}

The Human Development Index (HDI) was created to drive attention, in the evaluation of development, to aspects overshadowed by economic variables like the Gross Domestic Product (GDP) and its variants. In the computation of HDI, health and education conditions, directly accessed by longevity and schooling evaluations, are combined with a per capita income index to produce a unique score. There is a natural difficulty in combining different dimensions in a global score and different rules combining health, education, and income components have been tried in different moments (UNDP, 2016).

To be more efficiently employed to drive actions to reduce inequality between countries, the index should consider other dimensions in addition to those present in its initial formulation.

*Corresponding author.

ESG - Escola Superior de Guerra, Av. João Luiz Alves, s/n - 22291-090 Urca, Rio de Janeiro, RJ, Brazil.

E-mails: annibal.parracho@gmail.com; luiz.gaviao67@gmail.com 
Claims for the inclusion of the political dimension, involving the democratic freedoms, the legal safety, and the protection of discriminated groups, have been heard since the first years of computation of the Index (Haq, 1995; Sen, 2001; Klugman, Rodriguez \& Choi, 2011; Gurgel \& Mota, 2013). The aspect of sustainability, considering the interests of future generations, mainly affected by the environmental dimension and the need of preservation of natural resources, is also missing (Neumayer, 2001). During the last decade, other important improvements, adding new dimensions, like technology and culture, were suggested by Kovacevic (2010) and MayerFoulkes (2010).

Among all dimensions, those considering the inequality in assessing goods and services are those of main concern. The per capita income does not consider the intensity of poverty that stems from income inequality in the access to development. Inequalities in health and education are also not considered in the respective aggregate indices. Recently, a corrected HDI to consider inequality was included in the Human Development Report (UNDP, 2015). In this inequality adjusted HDI, a component measuring inequality in longevity, schooling and income modifies the basic index.

Another important aspect is gender inequality (Ferrant, 2014; Klasen, 2006; Permanyer, 2013). Different forms to access it have also been contemplated in the successive annual reports, leading to a set of four dimensions where the measurement of inequality is present in UNDP (2015).

In addition to lack these important dimensions of human well being, even in the dimensions taken into account, each component of the index evaluates the development only imprecisely. In Sant'Anna (2015), the ability of the Composition of Probabilistic Preferences (CPP) in considering the measurements imprecision is explored. Using CPP, the components of HDI are combined through probabilities of joint occurrence. This allows also to eliminate the need of employing ad hoc methods to get different dimensions comparable.

Here CPP is employed to combine, in parallel with the four initial components, four inequality evaluation ones. To assign higher importance to the effort to escape poverty, the CPP scores generated are based on the probability of not reaching the frontier of worst behavior in the measurement of any of its eight components.

The probabilistic scores so obtained are used not only to directly rank the countries but also to determine a Choquet capacity (Choquet, 1953) to be used, as in Merad et al. (2013), in subsequent compositions. One such application of the Choquet capacity consists in developing a CPP-Tri (Sant'Anna et al., 2015) procedure to classify the 188 countries in homogeneous classes.

\section{METHODOLOGICAL ISSUES}

\subsection{The Ranking Procedure}

CPP is a methodology designed to take into account, in the composition of decision criteria, the presence of imprecision. By treating the evaluations as observations of random variables, it generates rules, based on probabilities of choice to rank options evaluated by different criteria or different judges. Its first step consists in associating probability distributions to the evaluations 
of each option. The key idea is that each evaluation determines a value around which the measurements may vary. Instead of exact final preferences, the observed values are seen as location parameters of the probability distributions of possible values that would be assigned to the same option in other preference assessments under similar circumstances. In other words, the exact values of the decision matrix are turned into parameters of random variables.

The replacement of exact measurements by probability distributions enables to calculate the probability of each measure to be the preferred option according to each criterion. Applying proper composition rules, from these probabilities relative to each criterion global probabilities of preference may derive.

The simplest composition rule explores the basic concepts of conditional probabilities. The probabilities of being the best option according to each criterion are considered as conditional on preference for such criterion. Thus, an unconditional global preference is determined as a linear combination of these conditional probabilities. The preferences for the criteria enter as the marginal probabilities of the conditioning events.

An advantage of the probabilistic transformation is to give the evaluations according to different criteria the same form of probabilities. This allows to combine them through a Choquet integral with respect to a capacity, rather than by a weighted average as above suggested. The concept of capacity, developed by Choquet to extend the physical concept corresponding to the total charge a component can hold while maintaining a given potential energy, became an extension of the mathematical concept of measure (Choquet, 1986). In the context of multicriteria analysis, a capacity in a finite set of criteria $S$ is any nondecreasing function $C$ defined on the algebra of parts of $S$, satisfying the constraints of $C(\emptyset)=0$ and $C(S)=1$.

Using a Choquet integral with respect to a capacity instead of a weighted average corresponds to allowing for interaction between the criteria, instead of imposing additivity. In the capacity, the presence of positive interaction between two sets of criteria is revealed giving a higher value to the union of the sets and the presence of negative interaction by giving a lower value.

To evaluate the level of interaction, Shapley (1953) values may be used. The Shapley value of a criterion expresses its relative importance in the decision problem, i.e. its power to make alone the decision regardless the remaining criteria (Grabisch \& Roubens, 2000). The Shapley value of criterion $c$ of the set of criteria $S$ in the capacity $C$ in $S$ is given by Shapley

$$
(C, c)=\sum K \subset S \backslash\{c\}((\#(S \backslash\{c\} \backslash K)) !(\#(K)) ! /(\#(S)) !)(C(K \cup\{c\})-C(K)) .
$$

If a criterion tends to present positive interaction with other criteria its Shapley value will be higher than its individual capacity. The opposite will happen if it tends to interact negatively.

The Choquet integral $I_{C}(f)$ of the function $f$ defined on the set $S=\left\{c_{1}, \ldots, c_{s}\right\}$ with respect to the capacity $C$ on $S$ is obtained in the following way; first determine a permutation $\left\{c_{(1)}, \ldots, c_{(s)}\right\}$ of $S$ such that $f\left(c_{(1)}\right) \leq f\left(c_{(2)}\right) \leq \ldots \leq f\left(c_{(s)}\right)$ and set $f\left(c_{(0)}\right)=0$; then,

$$
I_{C}(f)=\sum_{k \in\{1, \ldots, s\}}\left(f \left(c_{(k)}-f\left(c_{(k-1)}\right) C\left(\left\{c_{\{k\}}, c_{(k+1)}, \ldots, c_{(s)}\right\}\right) .\right.\right.
$$


Equivalently,

$$
I_{C}(f)=\sum_{k \in\{1, \ldots, s\}} f\left(c_{(k)}\left(C\left(\left\{c_{\{k\}}, c_{(k+1)}, \ldots, c_{(s)}\right\}\right)-C\left(\left\{c_{(k+1)}, \ldots, c_{(s)}\right\}\right)\right) .\right.
$$

This definition makes the value of the integral higher when the value given by one of two criteria is high; it is also higher if there is positive interaction between the criteria than if it is negative.

A third form of composition permitted by the probabilistic transformation consists in employing probabilities of unions and intersections. To compute these probabilities, hypotheses of independence and of maximal dependence may be assumed. Maximal dependence leads to calculate the probability of the intersection by the minimum of the probabilities of the events intersecting and the probability of the union by the maximum, in a form of composition equivalent to the application of the principles of necessity and sufficiency of Fuzzy Logic (Zadeh, 1999). Independence allows to use the product where maximal dependence would use the minimum, enhancing robustness. The importance of the dependence in particular contexts can be assessed comparing by the joint probabilities computed under the two hypotheses.

Four variants of this last form of composition are studied in Sant'Anna (2015), exploring the extreme positions in conservatism and pessimism axes. In the first axis, the decision maker in the progressive side of the axis considers the probabilities of maximizing the preference according to the criteria, while that in the conservative side prefers to consider the probabilities of not minimizing it. The progressive decision maker pays attention to distances to the extremes of excellence, while the conservative one pays attention to distances to the extreme of worst performance. On the other hand, in the optimistic-pessimistic axis, the optimistic extreme is to consider satisfactory being the best in at least one criterion or not being the worst by all of them. The global score is then determined by the probability of maximizing preference according to at least one among the multiple criteria or not minimizing it according to all of them. Alternatively, in the pessimistic extreme, preference is measured by the probability of maximizing the preference according to all criteria or not minimizing it with respect to any of them.

In the present case, a reason to take a conservative approach is given by the priority to maximize the distance from extreme poverty and a reason to take a pessimistic approach by the need to avoid that extreme in every dimension of development.

\subsubsection{Algorithm for Capacity Estimation}

The computation of these joint probabilities may be used in a preliminary stage of composition by the Choquet integral. If there is a possibility of interaction between the criteria in the determination of the preferred option, such interaction may be assessed by comparing the maxima of the preferences along the options for the criteria taken together and isolated. In Sant'Anna (2015), this idea is developed to build an estimation procedure for the capacity driving the combination of the criteria. The capacity of each set is made proportional to the maximum along the options of the probabilities of being preferred according to at least one of the criteria in the set. 
The constant of proportionality depends on the maximal number q of sets with interaction taken into account in the computation of the integral. Formally, the capacity of a set $A=\left\{A_{1}, \ldots, A_{m}\right\}$ of a number $m \leq q$ of criteria will be given by $C(A)=\max _{j \in\{1, \ldots, n\}}\left(1-\prod_{k \in\{1, \ldots, m\}}(1-\right.$ $\left.\left.P_{k j}\right)\right) / M$, where $n$ is the number of options, $P_{k j}$ is the probability of option $j$ being the preferred by the $k$-th criterion among all options and $M$ is the maximum of $C(A)$ for $A$ varying along the sets of $q$ criteria. For sets of more than $q$ criteria, the capacity is set equal to 1 .

Small values of $q$ make easier the interpretation of the results, with the counterpart of biasing the final scores by subestimating the possible interaction in sets of a larger number of elements.

\subsection{The Classification Procedure}

In the classification approach, instead of ranking the options, the goal is to allocate each option in one of a set of ordered classes, previously determined. In CPP-Tri, the classes are determined by representative profiles.

To introduce the methodology in detail, we may consider each option separately. Let us denote this single option assessments according to the $s$ criteria by a vector $\left(a_{1}, \ldots, a_{s}\right)$ of $R_{s}$. Let us denote by $C=\left\{C_{1}, \ldots, C_{r}\right\}$ the set of $r$ classes, ordered in such a way that higher indices correspond to better options. Each class is represented by a representative profile, which is a vector of $R_{S}$ whose coordinates are typical evaluations by the $s$ criteria of an option belonging to such class. In the present application, each class will be represented by a unique profile. Let us denote by $\left(A_{i 1}, \ldots, A_{i s}\right)$ the representative profile of the $i$-th class.

The classification rule depends on the property of the probabilities of the evaluations of the option being above the profiles of a class with a higher index not being larger than the probabilities of being above those of a class with a smaller index while, inversely, the probabilities of being below are not smaller. Formally, this property holds if the profiles satisfy the non-crossing condition: if $i_{1}<i_{2}$, then $A_{i 1 k} \leq A_{i 2 k}$ for all $k$ from 1 to $m$.

The classification procedure runs as follows. Derive from each $a_{k}$ a random variable $X_{k}$ measuring the preference for the option by the respective criterion. Denote by $A_{i k}^{+}$and $A_{i k}^{-}$, respectively, the probability of $X_{k}$ to assume values above and below the coordinate $A_{i k}$ of the representative profile of the $i$-th class:

$$
A_{i k}^{+}=P\left[X_{k}>A_{i k}\right] \text { and } A_{i k}^{-}=P\left[X_{k}<A_{i k}\right] .
$$

The rule for the composition of global scores $A_{i}^{+}$and $A_{i}^{-}$of position in relation to the $i$-th class is based on the joint probability of the option being, respectively, above and below the class profiles according to all criteria. Assuming independence between the coordinates Xk corresponding to different criteria, the final scores will be $A_{i}^{+}=\prod_{k} A_{i k}^{+}$and $A_{i}^{-}=\prod_{k} A_{i k}^{-}$. The final step consists in locating the option in the class $i$ with the smallest absolute value for the difference $A_{i}^{+}-A_{i}^{-}$.

Ties may happen and the option may be indifferently located in contiguous classes. However, with continuous distributions and suitable numerical precision, an option receiving the same score for different classes will be rare. 
As in the case of ranking, in CPP-Tri other composition rules may be applied, instead of that based on joint probabilities. To take into account the possibility of interaction between the criteria, Choquet integrals may be used to combine the $A_{i k}^{+}$and the $A_{i k}^{-}$. In the present application, the capacity derived by maximizing preferences as described in the preceding section will be used in this form of composition.

In principle, the a priori determination of classes by means of the representative profiles is required. In practice, it is not necessary. This is due to the fact that, in practice, not one option only, but many options are classified. Representative profiles of the classes can be derived from the observation of the values assigned to the different options. A typical profile for the $i$-th of $r$ classes may be the vector whose $k$-th coordinate is the quantile of order $(2 i-1) /(2 r)$ of the set of evaluations of the options according to the $k$-th criterion. For example, to determine five classes, the coordinates of the representative profiles will be formed, respectively, by the values of the first, third, fifth, seventh and ninth deciles of the set of evaluations by each criterion. Analogously, in the case of nine classes, the nine deciles will be used.

This way to build representative profiles may result in vectors of profiles very different from those actually observed in the joint assessment of the options by the set of criteria. In fact, options with vectors of evaluations close to those found in the profiles formed by extreme quantiles, with very low or very high ratings by all the criteria, are rarely found. A slightly different strategy will be employed here to generate more realistic profiles. Instead of seeking quantiles of order $(2 i-1) /(2 r)$ in the ordering by each criterion isolated, it consists of selecting as representative profile the whole vector of evaluations of the option placed in this position in a global ranking.

A problem with this last strategy is that, unlike the profiles generated reaping the quantiles of distributions on isolated criteria, these vectors may not serve as representative profiles if they do not satisfy the non-crossing condition. The elements of the vector of evaluations of a country ranked in a position higher than another need not be all better than those of the other. If that happens, corrections must be applied wherever such prohibited crossing occurs. The following inversion correction algorithm is used here to obtain profiles without inversions but still close to those vectors of real evaluations initially selected.

\subsubsection{Algorithm for Correction of Inversions}

Denote by $Q_{i k}$ the correction for the coordinate $A_{i k}$.

If the number $r$ of classes is an odd number, keep unchanged the central class, that of order $(r+1) / 2$. If $r$ is even, replace the coordinates of the profiles of the classes of order $r / 2$ and $r / 2+1$ that present inversions by their arithmetic mean. That means, if $A_{r / 2 k}>A_{r / 2+1 k}$, then $Q_{r / 2 k}=Q_{r / 2+1 k}=\left(A_{r / 2 k}+A_{r / 2+1 k}\right) / 2$.

For those classes $i$ satisfying $1<i<r / 2$ (that means, for those classes whose profiles must be smaller than those of the central classes, except for the first), make the following corrections in a decreasing order: 
1) if $Q_{i+1 k}<A_{i k}$, then: if $A_{1-1 k}>Q_{1+1 k}$, then $Q_{1 k}=Q_{1+1 k}$; otherwise, $Q_{i k}=\left(A_{i-1 k}+\right.$ $\left.Q_{i+1 k}\right) / 2$; and

2) if $Q_{i+1 k}=A_{i k}, Q_{i k}=A_{i k}$.

Analogously, for the classes $i$ satisfying $(r+1) / 2<i<r$, (that means, for those classes whose profiles must be larger than those of the central classes, except for the last one), in an increasing order:

1) if $Q_{i-1 k}>A_{i k}$, then: if $A_{1+1 k}<Q_{1-1 k}$, then $Q_{1 k}=Q_{1-1 k}$; otherwise, $Q_{i k}=\left(A_{i+1 k}+\right.$ $\left.Q_{i-1 k}\right) / 2$; and

2) if $Q_{i-1 k} \leq A_{i k}, Q_{i k}=A_{i k}$.

For the first class: if $Q_{2 k}<A_{1 k}$, then $Q_{1 k}=Q_{2 k}$; otherwise, $Q_{1 k}=A_{1 k}$.

For the last class, if $Q_{r-1}>Q_{r}$, then $Q_{r k}=Q_{r-1 k}$; otherwise, $Q_{r k}=A_{r k}$.

\section{THE MODEL WITH INEQUALITY COMPONENTS}

\subsection{The Components of the Index}

In its last computations (UNDP, 2015; 2016), the following indices were used to compose HDI: life expectancy at birth, with limits of 20 and 85 years (UNDESA, 2015), the natural logarithm of gross national income per capita, with limits of 100 and 75000 dollars, adjusted according to the 2011 PPP (WORLD BANK, 2016) and, as education indicator, the arithmetic mean between two educational measures: the average number of years that a 25 -year-old person at present attended school (Barro \& Lee, 2016) and the expected value of the number of years that a six-year-old person at present will attend school (UNESCO, 2016). This second parameter is estimated from the current distribution of school enrollment raised in national censuses and surveys and employing the estimation methodology of Barro \& Lee (2016). These last two indicators are standardized to values between zero and one, by dividing the first by 15 and the second by 18 . The other two are standardized by subtracting the minimum and dividing by the range. To these four components, four indicators of reduction of inequality are here added: the life expectancy, education and income inequality indicators of UNDP and its Gender Development Index (UNDP, 2016).

The three components relative to inequality in the distribution of HDI dimensions are also extracted from the 2015 and 2016 Human Development Reports. In these reports, following Atkinson (1970) proposal with aversion parameter e equal to 1, equality is measured by the ratio between the geometric mean and the arithmetic mean of the distribution. These means are calculated for distributions obtained using the life tables provided by UNDESA (2015) in the case of life expectancy, household survey data in the case of schooling and for the income, disposable household income or consumption per capita data from the same international databases and household surveys. For a few countries, the last was obtained from an asset index matching methodology (Harttgen \& Vollmer, 2011). Income per capita outliers - extremely high incomes 
as well as negative and zero incomes - were dealt with by truncating at the top 0.5 percentile of the distribution and by replacing the negative and zero incomes by the minimum positive income.

UNDP publishes also a Gender Inequality Index that incorporates in the analysis a set of variables measuring concepts such as adolescent birth rate, share of parliament seats, attainment of educational levels and labour market participation. To keep the measurement of inequality restricted to the concepts composing the HDI, in this work the Gender Development Index (GDI) has been used in place of the Gender Inequality Index. GDI is calculated as the ratio between a female HDI and a male HDI, both determined by composing life expectancy, mean and expected schooling, and per capita income indices for each gender as in the composition of the general HDI. Note that the computation of life expectancy indices by gender takes into account the average of five years of biological advantage of women over men. This results in using for women limits of 22.5 and 87.5 years and for men limits of 17.5 and 82.5 years.

It was not possible to compute all four last indicators for all 188 countries for which the HDI was computed. Thus, the missing data were replaced by the minimum observed for the same component. The meaning of this assignment rule may be read as relating not measuring inequality in some dimension to disregarding the presence in the country of inequality in that dimension. This may be unfair to countries whose missing values are due to collecting difficulties not related to high inequality. The consequences of this assumption are discussed, below, in the analysis of the obtained scores. A full account of the data sources and procedures used in these estimations is available at: http://hdr.undp.org/en/statistics/ihdi/.

\subsection{The Model}

The application of CPP starts by treating the observed values of the HDI components as location parameters of random variables. Following the practice adopted in the construction of HDI, that is to constrain the indicators within 0 to 1 , the recorded assessments for each country in each indicator are treated as modes of triangular random variables with distributions varying from 0 to 1. In a conservative approach, this implies the presence of asymmetry in the evaluation process, the higher values more probably incorporating positive disturbances than the smaller ones.

In Sant'Anna et al. (2015), for the distribution of the HDI components in the range [0,1], beta distributions were used with parameters estimated on the basis of observations of a sequence of nine years. With the inclusion of the four components measuring inequality, only available by 2015 , there are not enough data to apply this approach. Beta and triangular distributions, in addition to bounding variation to the interval $[0,1]$, are both asymmetrical, assigning higher probability to drifts downward in the case of high modes and upwards otherwise. In the case of HDI, where the values of the components are becoming higher over time, this produces an automatic adjustment toward lower scores.

In the initial procedure to rank the countries, to compose the probabilities of preference by the different criteria, a pessimistic conservative point of view is taken. This is justified by the priority of the aim of moving away from the worst situations and to the idea that this objective should 
equally apply to all dimensions. To generate the global score for each option, this point of view results in employing the joint probability of not presenting the lowest evaluation according to any criterion.

To compute the probabilities, independence between distributions is initially assumed. Thus, the score for each particular country is obtained as the product, along the eight components, of the probabilities of the value of the indicator for that country not being the lowest among the 188 values for it. After the joint probabilities are computed, they can be used to derive a capacity and the composition at the classification stage employs a Choquet integral with respect to the so derived capacity. This corresponds to a relaxation of the assumption of independence, justified by the fact that the inequality measurements partially involve repetition of the calculations of the basic components.

The capacities employed were derived from the probabilities of minimization of preferences as described in Section 2.2, admitting the interaction between criteria up to a given number $q$. The capacities of the sets increase, as expected, with the value of $q$, but, otherwise, do not change considerably. In these capacities, it is relevant the importance given to the average years of schooling. Among the unitary sets, the highest capacity, of 0.49 for $q=4$, is for this criterion. Among the binary sets, the highest is 0.69 , for the set formed by expected and average years schooling. On the other end, the unitary set with the smallest capacity is education inequality, with a capacity of 0.14 , and the binary set with the smallest capacity is formed by education inequality and the gender development index, with a capacity of 0.29 .

In fact, for all values of $q$, the higher importance, among the eight criteria, is given to the components of HDI related to education. They receive Shapley values around 0.16 and 0.24 , respectively, in the first year, and around 0.14 and 0.27 in the second, against Shapley values around 0.1 for all other six criteria. The approaches based on previously assigning weights to balance the importance of the different dimensions would not allow for detection of differences like these.

Modeling the uncertainty with an asymmetric distribution makes difficult to anticipate which options will fall in each class, because the probabilities of being above and below the profile will be different for asymmetric distributions with the mode in a vector of values near the profile, shifting to the center. Then, to grant discrimination, a large number of broadly spaced initial classes is advisable. In the present application, where in the HDR a division of the countries in four classes is employed, this led to the use of an initial number of nine classes with their representative profiles spread over the whole range of possible evaluations.

Representative profiles for the nine classes are then built on the basis of the initial evaluations of the countries positioned at the ranks 19, 38, 57, 76, 113, 132, 151 and 170 and, for the central class, the arithmetic mean between those of the countries ranked as 94th and 95th. These profiles for the first year are shown in the columns headed by "Initial" in Table 1. The application of the algorithm of Section 2.2.1 to correct the cases where inversions are observed in these initial profiles eventually leads to the set of profiles shown in the columns headed by "Corrected" in Table 1. It can be seen in Table 1 that the main inversions are between values of classes 1 and 2 
and of classes 7 and 8 . It can also be seen there that a large majority of initial values is preserved and the vectors of profiles obtained are clearly distinct.

Table 1 - Profiles for the Nine Classes.

\begin{tabular}{|c|c|c|c|c|c|c|c|c|}
\hline Criterion & \multicolumn{2}{|c|}{ Longevity } & \multicolumn{2}{|c|}{$\begin{array}{l}\text { Expected } \\
\text { Schooling }\end{array}$} & \multicolumn{2}{|c|}{$\begin{array}{c}\text { Mean } \\
\text { Schooling }\end{array}$} & \multicolumn{2}{|c|}{ Income } \\
\hline Class & Initial & Corrected & Initial & Corrected & Initial & Corrected & Initial & Corrected \\
\hline 1 & 0.62 & 0.50 & 0.52 & 0.50 & 0.22 & 0.22 & 0.44 & 0.44 \\
\hline 2 & 0.50 & 0.50 & 0.50 & 0.50 & 0.40 & 0.40 & 0.60 & 0.49 \\
\hline 3 & 0.84 & 0.66 & 0.78 & 0.56 & 0.51 & 0.41 & 0.74 & 0.54 \\
\hline 4 & 0.84 & 0.82 & 0.82 & 0.61 & 0.48 & 0.43 & 1.00 & 0.54 \\
\hline 5 & 0.82 & 0.82 & 0.61 & 0.61 & 0.43 & 0.43 & 0.54 & 0.54 \\
\hline 6 & 0.83 & 0.83 & 0.75 & 0.75 & 0.49 & 0.49 & 0.72 & 0.72 \\
\hline 7 & 0.89 & 0.89 & 0.66 & 0.87 & 0.62 & 0.62 & 0.69 & 0.77 \\
\hline 8 & 0.87 & 0.89 & 1.00 & 1.00 & 0.66 & 0.66 & 0.82 & 0.82 \\
\hline 9 & 0.87 & 0.89 & 0.92 & 1.00 & 0.83 & 0.83 & 0.84 & 0.84 \\
\hline Criterion & \multicolumn{2}{|c|}{$\begin{array}{l}\text { Longevity } \\
\text { Inequality }\end{array}$} & \multicolumn{2}{|c|}{$\begin{array}{l}\text { Education } \\
\text { Inequality }\end{array}$} & \multicolumn{2}{|c|}{$\begin{array}{c}\text { Income } \\
\text { Inequality }\end{array}$} & \multicolumn{2}{|c|}{ G D I } \\
\hline Class & Initial & Corrected & Initial & Corrected & Initial & Corrected & Initial & Corrected \\
\hline 1 & 0.66 & 0.59 & 0.55 & 0.50 & 0.89 & 0.32 & 0.60 & 0.60 \\
\hline 2 & 0.59 & 0.59 & 0.57 & 0.50 & 0.72 & 0.32 & 0.84 & 0.72 \\
\hline 3 & 0.83 & 0.83 & 0.50 & 0.50 & 0.32 & 0.32 & 0.84 & 0.84 \\
\hline 4 & 0.93 & 0.84 & 0.50 & 0.50 & 0.32 & 0.32 & 0.97 & 0.88 \\
\hline 5 & 0.86 & 0.86 & 0.77 & 0.77 & 0.77 & 0.77 & 0.93 & 0.93 \\
\hline 6 & 0.86 & 0.86 & 0.79 & 0.79 & 0.63 & 0.79 & 1.00 & 1.00 \\
\hline 7 & 0.90 & 0.90 & 0.88 & 0.88 & 0.82 & 0.82 & 0.95 & 1.00 \\
\hline 8 & 0.91 & 0.91 & 0.92 & 0.92 & 0.74 & 0.82 & 0.98 & 1.00 \\
\hline 9 & 0.94 & 0.94 & 0.98 & 0.98 & 0.81 & 0.82 & 1.00 & 1.00 \\
\hline
\end{tabular}

\section{DISCUSSION}

Except the worsening of the rank of those countries for which it was not possible to obtain an evaluation for some inequality component, few large changes with respect to HDI ranks without inequality adjustments are observed in the results obtained by the application of the conservative pessimistic CPP approach. The most noticeable falls in ranks with respect to those derived from HDI without inequality adjustment occur in the two years for Singapure and Liechsteinstein, countries with HDIs among the 15 highest, and the most noticeable improvements for the republics of the former Soviet Union of Moldova and Tajikistan. In both years, African countries occupy the final 20 ranks and the lowest class by the different forms of composition, except for the presence of the countries of Yemen, and Afghanistan, countries enduring civil war. 
The classification obtained for the last year, employing the capacities derived from the probabilities of minimization applying an $\mathrm{R}$ (R-Core-Team, 2016) procedure developed on purpose and the package kappalab (Grabisch, Kojadinovic \& Meyer, 2008) is presented in Table 2, for $q=8$. The $\mathrm{R}$ code is presented in Appendix 1.

Modeling with asymmetric triangular distributions, by offsetting the concentration of indicators in high values, produced the emptying of the classes represented by the profiles derived from the highest and the lowest deciles. Four classes, the same number used in the UNDP reports to divide the countries according to HDI, are still nonempty, even though the increase in the number of dimensions blends the countries and hinders discrimination. The names of the countries in classes 3 and 4 are preceded in Table 2 by a letter denoting its classification in the 2016 HDR: V, for Very High, H, for High, M, for Median, and L, for Low, to call attention to the effect of taking inequality into account. All countries in Class 2 are in the Low Development class in HDR and all countries in Class 5, except Belarus, are in the Very High Development class. All countries in Class 3 are in the Low or Median Development classes in HDR except Dominica, which is in the High Development Class. All countries in Class 4 are in the Median, High or Very High Development in HDR, with a clear dominance of High.

The results in Table 2 are similar to those obtained if interactions are not taken into account, with all the countries being classified by the different rules in the same classes or in contiguous classes. The same happened in the first year. The main quality of the methodology here developed, its power to extract information from the data by means of free comparison of the evaluations, leads, in this case, to a concentration of countries in Class 4.

The capacity generated was also used afterwards to compose new scores by Choquet integrals. In such composition by Choquet integrals the replacement by the minimum will affect considerably less the ranks of the countries with missing values. This implies that considering the interactions reduced the influence of one or more inequality criteria.

Nevertheless, it must be pointed out that the differences between the results by the two ranking rules are not large, corresponding to high rank correlation coefficient (Spearman, 1904) for all values of $q$. The correlation coefficient with the HDI ranking is above 0.9 for all forms of composition in both years.

\section{CONCLUSION}

The probabilistic composition takes into account the imprecision in the measurement of development indicators. This allowed here to combine the indicators by a rule naturally derived from emphasizing distance to worst performance in all dimensions. Another composition rule was derived from this first one by allowing for interaction. The possibility of interaction between up to eight components was considered. Both these rules neither involve weighting the components nor transformation of the original measurement other than the transformation into probabilities.

The main feature of the new index, besides the homogeneity due to the treatment of the components in probabilistic terms and the prioritization of the distances to the frontier of lowest values, 
Table 2 - Classification of 188 Countries.

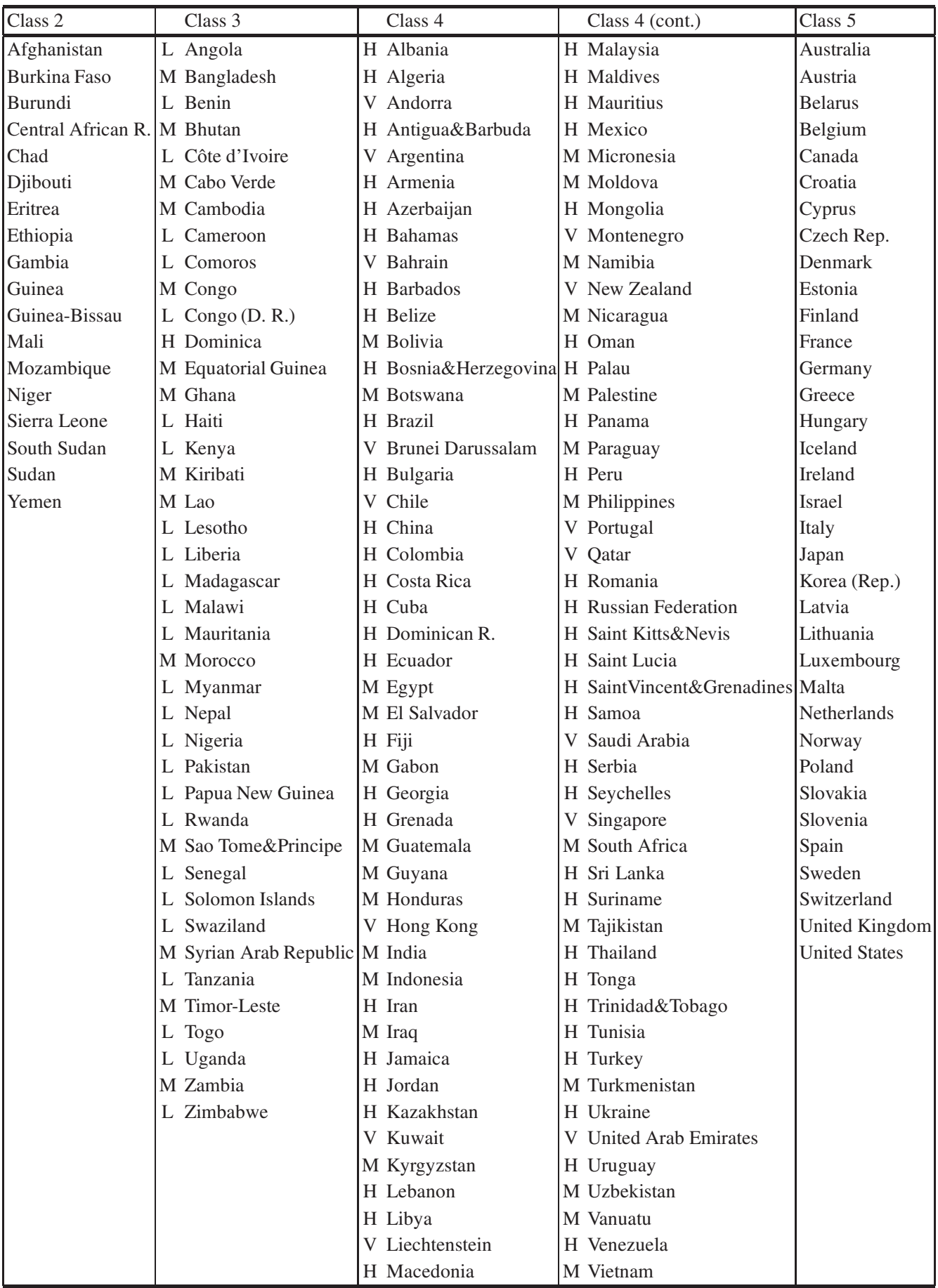


is the importance attached to inequality. To the original components of HDI four indicators of development in the direction of inequality reduction were added. Thus the new index directly combines eight components. This same structure of composition may be applied to include a larger set of dimensions in the index.

In addition to the ranking of countries in terms of probabilities of not reaching the extremes of worst evaluation, it was performed a classification in a limited number of ordered classes. This resulted in the classification of countries into four classes. For this application, an algorithm of building ordered representative profiles was produced. Again, automatic composition rules were employed based on simple probabilistic comparisons.

Missing data on the inequality dimensions were dealt with by assigning the smallest observed values in each component to the countries with no measurement available. This affects more the ranking based on the computation of joint probabilities. The other form of combination, by a Choquet integral with respect to the capacity obtained allowing for interaction between all the criteria, resulted in final ranks closer to those obtained without the addition of the four inequality dimensions. If classification is performed instead of ranking, the composition by joint probability and by the Choquet integral led to similar results.

\section{ACKNOWLEDGEMENTS}

We thank the reviewers for their careful reading and valuable comments, and CAPES for the financial support.

\section{REFERENCES}

[1] BARRo RJ \& LEE JW. 2013. A new data set of educational attainment in the world, 1950-2010. Journal of Development Economics, 104: 184-198.

[2] BilbaO-Ubillos J. 2013. Another approach to measuring human development: The composite dynamic Human Development Index. Social Indicators Research, 111(2): 473-484.

[3] Chaaban J, Irani A \& Khoury A. 2016. The Composite Global Well-Being Index (CGWBI): A New Multi-Dimensional Measure of Human Development. Social Indicators Research, 129(1): 465-487.

[4] Choquet G. 1953. Theory of capacities. Annales de l'institut Fourier, 5: 131-295.

[5] Choquet G. 1986. La naissance de la théorie des capacités: réflexion sur une expérience personelle. Comptes rendus de l'Académie des sciences. Série générale, La Vie des sciences, 3(4): 385-397.

[6] Ferrant G. 2014. The Multidimensional Gender Inequalities Index (MGII): A descriptive analysis of gender inequalities using MCA. Social Indicators Research, 115(2): 653-690.

[7] Grabisch M, Kojadinovic I \& Meyer P. 2008. A review of methods for capacity identification in Choquet integral based multi-attribute utility theory: Applications of the Kappalab R package. European Journal of Operational Research, 186(2): 766-785. 
[8] Grabisch M \& Roubens M. 2000. Application of the Choquet integral in multicriteria decision making. In: Grabisch M, Murofushi T \& Sugewno M. (eds.) Fuzzy Measures and Integrals - Theory and Applications, 348-374.

[9] Gurgel AM \& Mota CMM. 2013. A multicriteria prioritization model to support public safety planning. Pesquisa Operacional, 33(2): 251-267.

[10] HAQ M UL. 1995. Reflections on human development. Oxford University Press.

[11] Harttgen K \& Vollmer S. 2011. Inequality Decomposition without Income or Expenditure Data: Using an Asset Index to Simulate Household Income. Human Development Research Paper UNDP-HDRO, New York. http://hdr.Undp.Org/en/content/inequality-Decomposition-withoutIncome-or-Expenditure-Data.

[12] KLASEN S. 2006. UNDP's gender-related measures: some conceptual problems and possible solutions. Journal of Human Development, 7(2): 243-274.

[13] Klugman J, Rodriguez F \& ChOI HJ. 2011. The HDI 2010: new controversies, old critiques. The Journal of Economic Inequality, 9(2): 249-288.

[14] Kovacevic MA. 2010. Review of Critiques to HDI and Potential Improvements. Human Development Research Paper, 33.

[15] MAYER-Foulkes D. 2010. Divergences and convergences in human development. UNDP-HDRO Occasional Papers, (2010/20).

[16] Merad M, Dechy N, Serir L, Grabisch M \& Marcel F. 2013. Using a multi-criteria decision aid methodology to implement sustainable development principles within an organization. European Journal of Operational Research, 224(3): 603-613.

[17] Neumayer E. 2001. The human development index and sustainability - a constructive proposal. Ecological Economics, 39(1): 101-114.

[18] Permanyer I. 2013. A critical assessment of the UNDP's gender inequality index. Feminist Economics, 19(2): 1-32.

[19] R-Core-Team. 2016. R: A language and environment for statistical computing. http://www.RProject.Org., Vienna, Austria.

[20] Sant'Anna AP. 2015. Probabilistic Composition of Preferences, Theory and Applications. New York: Springer.

[21] Sant' Anna AP, Costa HG \& Pereira V. 2015. CPP-TRI: a sorting method based on the probabilistic composition of preferences. International Journal of Information and Decision Sciences, 7(3): 193-212.

[22] SEn A. 2001. Development as freedom. Oxford Paperbacks.

[23] Spearman C. 1904. The proof and measurement of association between two things. The American Journal of Psychology, 15(1): 72-101.

[24] UNDESA 2013. World Population Prospects database. New York. Retrieved from http: / / esa. un. org/unpd/wpp/.

[25] UNDP 2015. Human Development Report. Retrieveed from http://hdr.undp.org/sites/ default/files/2015_human_development_report.pdf. 
[26] UNDP 2016. Human Development Report. Retrieved from http: / / report. hdr . undp . org / .

[27] UNESCO 2015. Institute for Statistics, UIS Stat. Retrieved from http: / / s tat . uis . unesco. org.

[28] WORLD BANK 2015. PPP conversion factor (GDP) to market exchange rate ratio.

[29] Zadeh LA. 1999. Fuzzy sets as a basis for a theory of possibility. Fuzzy Sets and Systems, 100: 9-34.

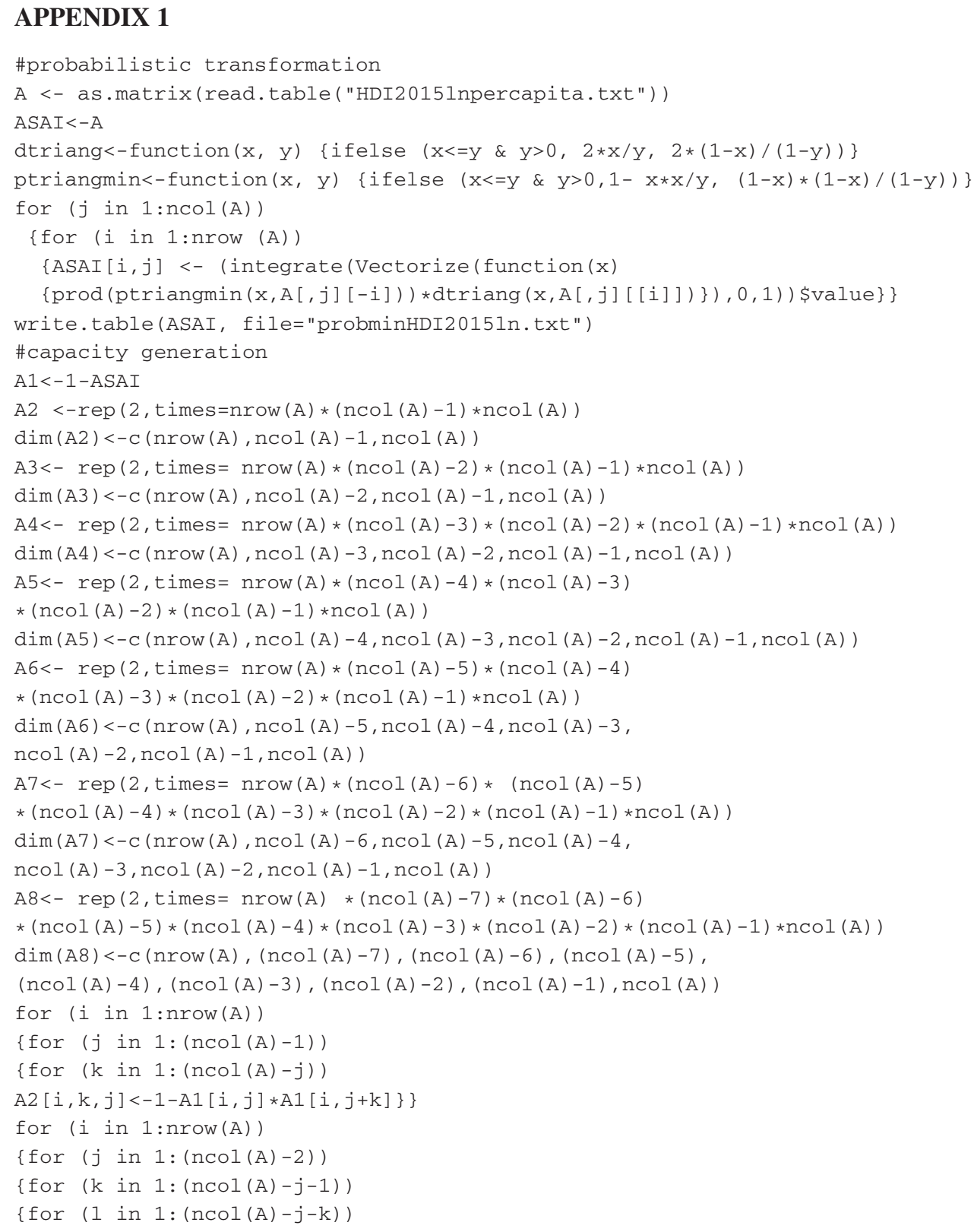




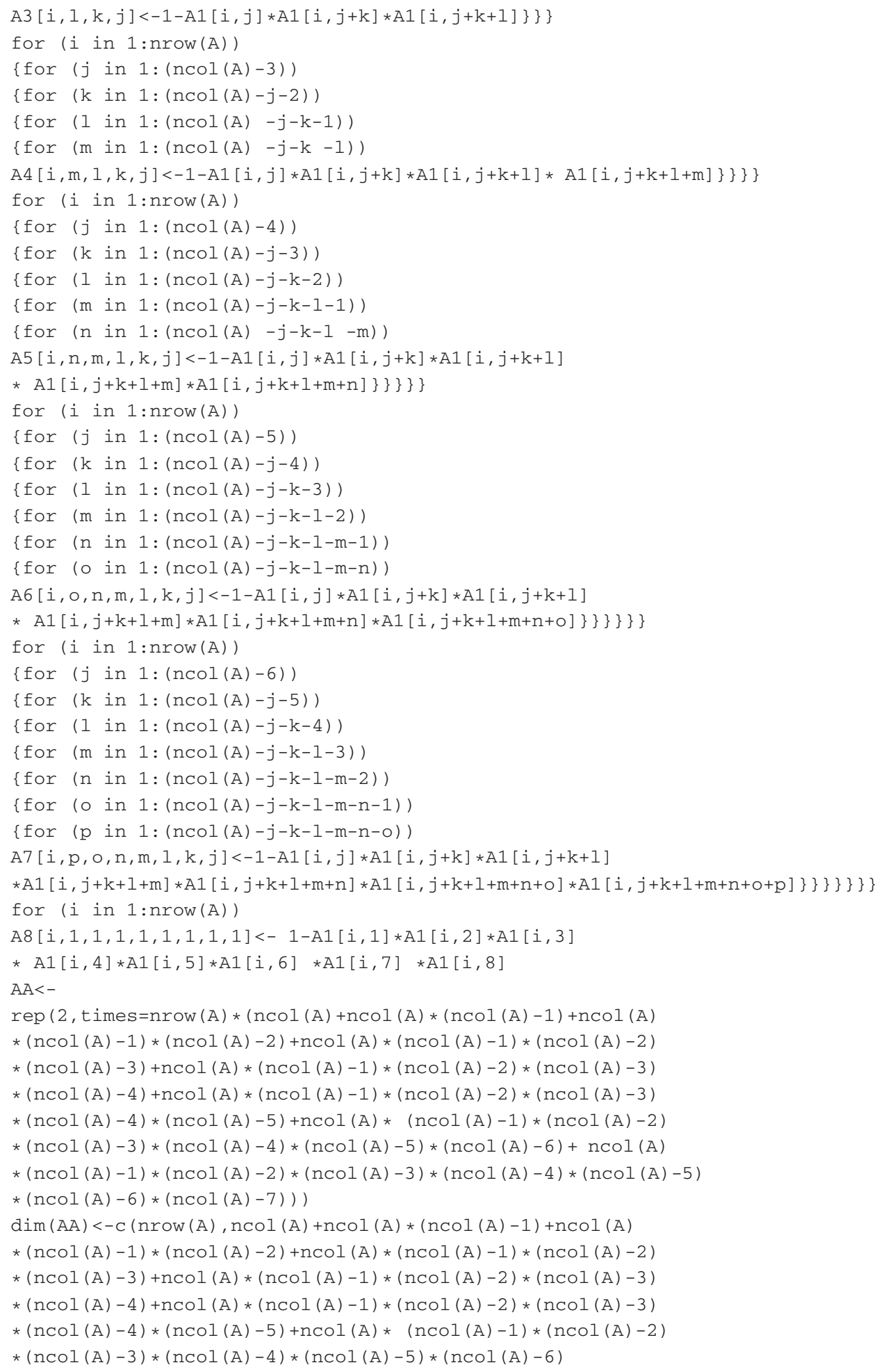




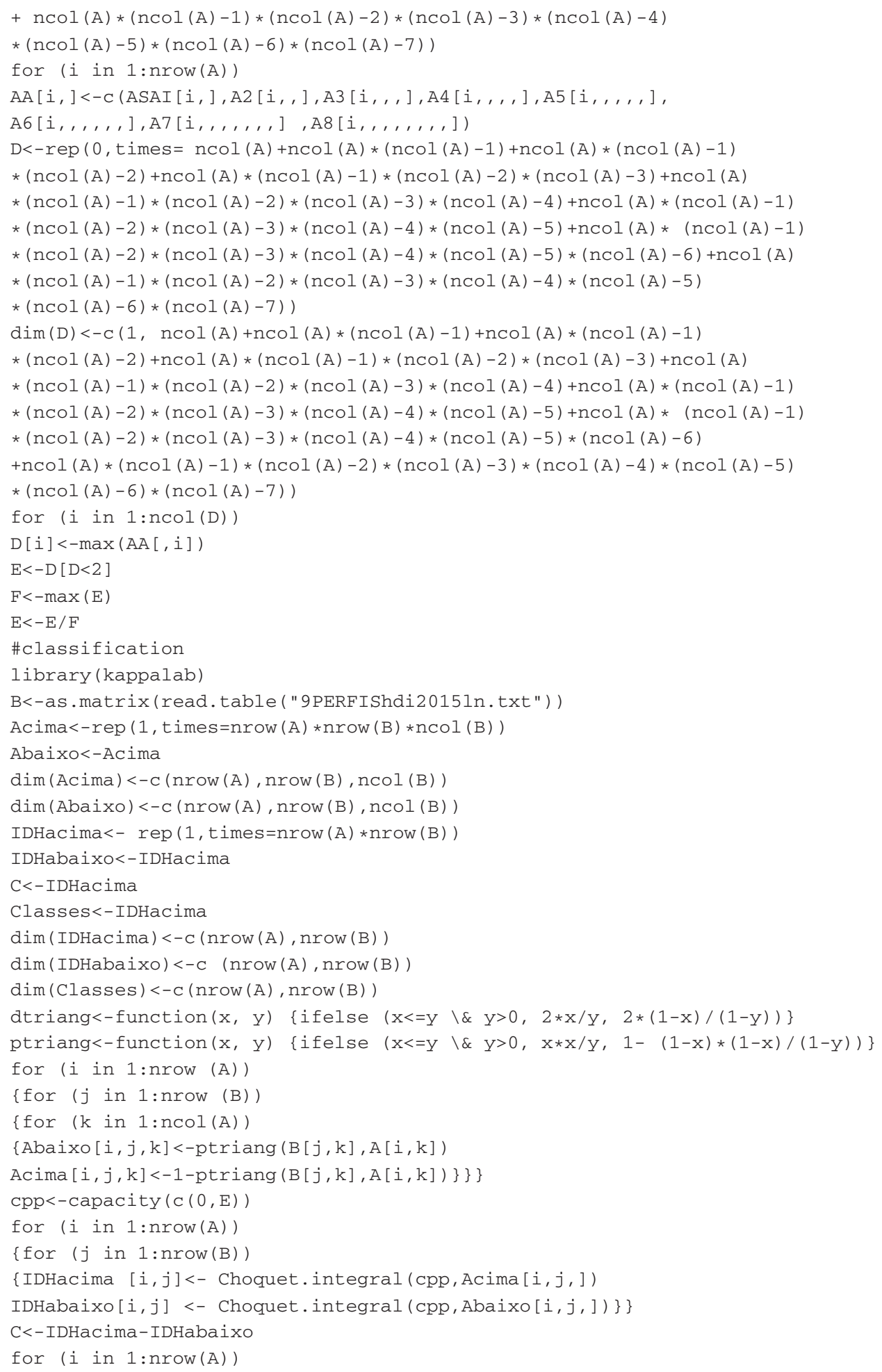




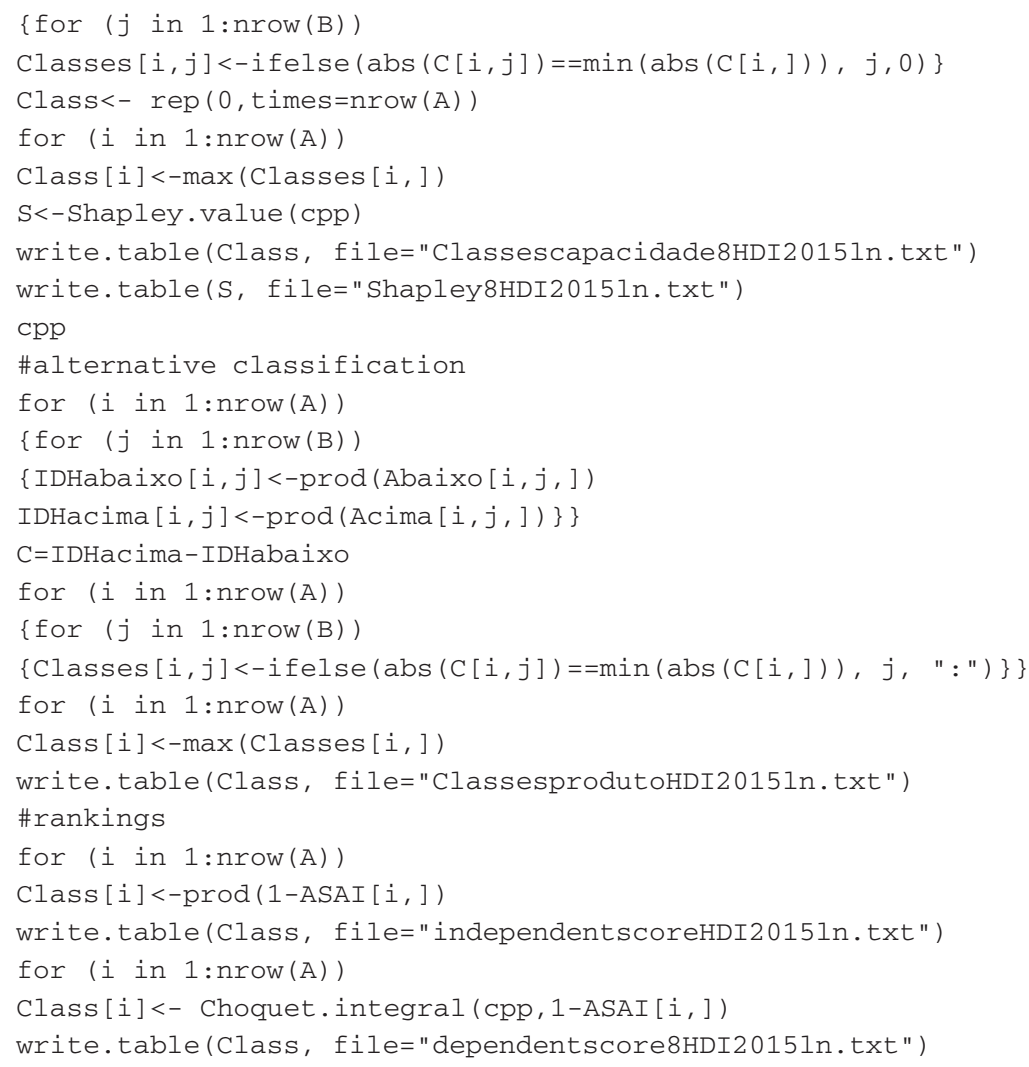

